Check for updates

Cite this: RSC Adv., 2018, 8, 15915

\title{
Sustainable synthesis of nanoporous carbons from agricultural waste and their application for solid- phase microextraction of chlorinated organic pollutants $\dagger$
}

\author{
Hu Cheng, ${ }^{a b}$ Yang Song, ${ }^{\text {ab }}$ Yongrong Bian, (D) *ab Rongting Ji, ${ }^{\text {ab }}$ Fang Wang, ${ }^{\text {ab }}$ \\ Chenggang Gu, ${ }^{\mathrm{ab}}$ Xinglun Yang ${ }^{\mathrm{ab}}$ and Xin Jiang*ab
}

To guarantee the safety of water resources for humans, there is a high demand for the development of highly-efficient probes for solid-phase microextraction and analysis of trace organic pollutants. In this work, we greenly synthesized nanoporous carbons (NPCs) from oilseed rape straw via a facile hydrothermal treatment and potassium bicarbonate activation. Results showed that the NPCs had partly graphitic, amorphous-like structures with a high surface area (up to $1253 \mathrm{~m}^{2} \mathrm{~g}^{-1}$ ), large pore volume (up to $0.71 \mathrm{~cm}^{3} \mathrm{~g}^{-1}$ ), high mesopore to total pore volume ratio (up to $29 \%$ ) and great thermal stability (>400 ${ }^{\circ} \mathrm{C}$ ). When the NPCs were utilized as a solid-phase microextraction fiber coating, the extraction efficiencies for chlorinated organic pollutants (COPs) were higher (1-38 times) than with a common commercial polydimethylsiloxane coating because of high surface adsorption energy, strong $\pi-\pi$ stacking interactions and large mass transfer capacity. Using the most efficient NPC- 8 coating, under optimum extraction conditions (desorption temperature, $290{ }^{\circ} \mathrm{C}$; extraction temperature, $80{ }^{\circ} \mathrm{C}$; extraction time, $25 \mathrm{~min}$ ), an analysis method for trace COPs in water was developed with good linearity (0.9991-0.9998), high sensitivity (limits of detections, $0.08-0.64 \mathrm{ng} \mathrm{L}^{-1}$ ), acceptable repeatability (RSDs of single fiber, $2.63-6.73 \%$ ) and great reproducibility (RSDs of fiber-to-fiber, $2.22-7.12 \%$ ). Finally, the NPC-8 coating was applied to a real environmental sample with satisfactory recoveries $(86.66-103.27 \%)$.

Received 10th March 2018 Accepted 24th April 2018 DOI: $10.1039 / c 8 \mathrm{ra02123f}$ rsc.li/rsc-advances ionic liquid, ${ }^{8,9}$ crosslinked polymer nanoparticle, ${ }^{10,11}$ molecularly imprinted polymeric, ${ }^{12,13}$ metal organic framework, ${ }^{\mathbf{1 4 , 1 5}}$ and carbonaceous material. ${ }^{16-18}$ Among those, nanoporous carbons (NPCs) have attracted more attention because of their huge specific surface area, large pore volume, excellent thermal stability and potential for $\pi-\pi$ interactions, hydrophobic interactions and dispersion forces to organic pollutants. ${ }^{19}$ To obtain NPCs, nanocasting (soft- and hard-templating) and activation $\left(\mathrm{KOH}, \mathrm{ZnCl}_{2}\right.$ or $\mathrm{FeCl}_{3}$ ) have always been used. ${ }^{20-23}$ Nanocasting is costly and complicated with multiple steps, while activation is low-cost and facile via one step. However, traditional activators, including $\mathrm{KOH}, \mathrm{ZnCl}_{2}$ and $\mathrm{FeCl}_{3}$, are corrosive which does harm to environment and limits the industrial production. To avoid these problems, an emerging green activator, potassium bicarbonate $\left(\mathrm{KHCO}_{3}\right)$, was chosen to synthesize NPCs for SPME. ${ }^{24}$ In addition, from the points of sustainability, economy and availability, crude biomass has been considered as ideal carbon precursor. Moreover, crude biomass could further develop the pore structure because of its multi-element composition. ${ }^{25}$

Agricultural waste, was generated in large amounts and remains after harvest every year, which have not only affected planting, but also increased environmental burden. ${ }^{26}$ Oilseed
${ }^{a}$ Key Laboratory of Soil Environment and Pollution Remediation, Institute of Soil Science, Chinese Academy of Sciences, No. 71 East Beijing Road, Nanjing 210008, P. R. China. E-mail: yrbian@issas.ac.cn; jiangxin@issas.ac.cn; Fax: +86 25 86881000; Tel: +862586881195

${ }^{b}$ University of Chinese Academy of Sciences, Beijing 100049, P. R. China

$\dagger$ Electronic supplementary information (ESI) available. See DOI: 10.1039/c8ra02123f 
rape is one of the leading feedstock for biofuel production. The area of land used for its cultivation has substantially increased recently, especially in Germany, France, Poland, China, and England, because of the renewable energy directive. In 2015, the National Bureau of Statistics reported that around 30 million tons of oilseed rape straw (OSRS) were generated after harvesting in China. ${ }^{27}$ However, there have been few reports on the recycling of OSRS as NPCs. Hence, in this study, OSRS was chosen as raw material and converted into high porosity carbon materials. The products are not only promising NPCs for highperformance SPME of organic pollutants, but could also be applied in the fields of gas storage, catalytic carrier, electron transfer and supercapacitor in the future.

Chlorinated organic pollutants (COPs), including chlorobenzenes (CBs) and polychlorinated biphenyls (PCBs), have been widely distributed in the global environment and seriously threaten human health because of their high toxicity, easy transfer and biological amplification. ${ }^{\mathbf{2 8 , 2 9}}$ To explore mechanisms of transfer, transformation and degradation, predict risks for human, it is necessary to develop the SPME technology to analysis trace levels of COPs.

Will the green activator, $\mathrm{KHCO}_{3}$, work for synthesizing expected NPCs from crop waste, OSRS? Does the prepared NPCs could be used as an efficient SPME fiber coatings for trace analysis of organic pollutants, such as COPs, in aqueous environments? Up to date, there is lacking report to answer these questions. In this study, we synthesized novel NPCs with large specific surface area and high pore volume by hydrothermal reaction ${ }^{30}$ and $\mathrm{KHCO}_{3}$ activation of OSRS. Then, silicone sealant was used to attach the NPCs to the surface of stainless steel wire. The homemade SPME coating was homogeneous and shown higher extraction capacities compared with commercial polydimethylsiloxane (PDMS) fiber toward COPs due to the developed porosity, hydrophobic and $\pi-\pi$ interaction. Under optimized extraction conditions, an accurate and sensitive method for analysis of twelve CBs and PCBs in water sample was developed. This work opened up new possibilities for sustainable conversion of agricultural waste into ideal NPCs for highperformance SPME, and provided a crucial foundation for the recycling of oilseed rape straw.

\section{Materials and methods}

\subsection{Substances and materials}

OSRS was collected from local farmland (Nanjing, Jiangsu, China). $\mathrm{KHCO}_{3}$ and cyclohexane were obtained from Sinopharm Chemical Reagent Co., Ltd (Beijing, China). CBs (1,3,5-trichlorobenzenes, 1,2,3-trichlorobenzenes, 1,2,3,4-tetrachlorobenzene, 1,2,3,5-tetrachlorobenzene, pentachlorobenzene and hexachlorobenzene), PCBs (2,4'-dichlorobiphenyl (PCB-8), 2,5-dichlorobiphenyl (PCB-9), 2,2',5-trichlorobiphe-nyl (PCB-18), 2,3,3'-trichlorobiphenyl (PCB-20), 2,4,4'-trichlorobiphenyl (PCB28) and 2,2',5,5'-tetrachlorobiphenyl (PCB-52)), chromatographic grade $n$-hexane and acetone were bought from Accustandard Inc. (New Haven, CT, USA). Stainless steel wire $\left(\begin{array}{lll}\Phi & 127 \mu \mathrm{m}\end{array}\right)$ was purchased from Small Parts (Miami, FL, USA). Silicone sealant was obtained from Sika Ltd. (HK, China). The PDMS fiber $(30 \mu \mathrm{m})$ and SPME manual holder were purchased from Supelco (Bellefonte, PA, USA). The empty SPME needle was obtained from XTrust Instruments (Shanghai, China).

\subsection{Synthesis of nanoporous carbons}

$10 \mathrm{~g}$ of dried OSRS were ground into powder and mixed with $40 \mathrm{~mL}$ ultra-pure water in the hydrothermal reactor for $4 \mathrm{~h}$ under $250{ }^{\circ} \mathrm{C}$. Subsequently, brown-black solid residue (hydrochar) was collected by filtration and dried in a conventional oven for $12 \mathrm{~h}$. Then, $\mathrm{KHCO}_{3}$ was mixed with hydrochar at a mass ratio of $0,2,4,8\left(\mathrm{KHCO}_{3} /\right.$ hydrochar, weight/weight $)$ in the beaker and denoted as NPC- $X$, in which $X=$ mixed mass ratio of $\mathrm{KHCO}_{3}$ to hydrochar. Carbonization and activation were followed by annealing the mixture at $850{ }^{\circ} \mathrm{C}$ with heating rate of $5{ }^{\circ} \mathrm{C} \min ^{-1}$ and hold for $1 \mathrm{~h}$ under $\mathrm{N}_{2}$ protection of 400 $\mathrm{mL} \mathrm{min}^{-1}$ in a TL1200 tubular furnace. The resulted black solid residue was washed with $1 \mathrm{M}$ hydrochloric acid to remove metallic compounds. Followed by dried at $120{ }^{\circ} \mathrm{C}$ for $12 \mathrm{~h}$, the novel and promising NPCs were obtained (Fig. S1†) ${ }^{24}$

\subsection{Fabrication of solid-phase microextraction fiber}

$127 \mu \mathrm{m}$ diameter of stainless steel wire was cut into about $2 \mathrm{~cm}$, and washed using ultrasound with acetone and ultra-pure water. After dried, cleaned wire was vertically immersed into cyclohexane-diluted silicone sealant, rolled and pulled out quickly. A thin film of silicone sealant was left on the surface of the stainless steel wire. Then, this wire was further rolled in the NPCs powder and conditioned at $90{ }^{\circ} \mathrm{C}$ in an oven. Repeating these steps twice to effectively avoid the pore blocking phenomenon that might be caused by the sol-gel technique. ${ }^{11,31,32}$

\subsection{Solid-phase microextraction procedure and gas chromatography analysis}

Before analysis, SPME fiber was conditioned at $250{ }^{\circ} \mathrm{C}$ for $2 \mathrm{~h}$ and installed in SPME manual holder. Then, exposed over the surface of water sample in $20 \mathrm{~mL}$ brown bottle, which was treated with water bath heating. Several minutes later, got SPME fiber out, and inserted into injection port of GC (Agilent 6890) at $290{ }^{\circ} \mathrm{C}$ for thermal desorption. Ultra-pure nitrogen (purity > 99.999\%) was used as carrier and make-up gas at constant flow rates of $2 \mathrm{~mL} \mathrm{~min}^{-1}$ and $20 \mathrm{~mL} \mathrm{~min}^{-1}$, respectively. An DB-5.625 capillary column $(30 \mathrm{~m} \times 0.32 \mathrm{~mm}$ i.d. $\times 0.25 \mu \mathrm{m})$ was used to separate twelve selected CBs and PCBs. Column temperature was programmed as follows: initial temperature $50{ }^{\circ} \mathrm{C}$, increased to $250{ }^{\circ} \mathrm{C}$ at $10{ }^{\circ} \mathrm{C} \mathrm{min}^{-1}$, then increased to $300{ }^{\circ} \mathrm{C}$ at $20^{\circ} \mathrm{C} \mathrm{m^{-1 }}{ }^{-1}$ and held for $2 \mathrm{~min}$. The electron capture detector (ECD) was used to analysis COPs. The total analysis time was 24.5 min.

\subsection{Structure characterization}

Thermogravimetric (TG) and differential scanning calorimetry (DSC) analysis were measured with PerkinElmer Thermal Analyzer. Scanning electron microscope (SEM) and transmission electron microscope (TEM) were performed to 
characterized morphology structure using a ZEISS EVO18 and JEM-2100 (HR), respectively. Nitrogen adsorption/desorption isotherms were measured at $-195.68{ }^{\circ} \mathrm{C}$ by means of a Micromeritics model ASAP 2020 sorptometer. X-ray diffraction (XRD) was performed with a SmartLab X-ray diffractometer operated at $45 \mathrm{kV}$ and $200 \mathrm{~mA}$. An InVia-Reflex apparatus was used to measure Raman spectrum with $532 \mathrm{~nm}$ laser. Fourier transform infrared (FTIR) spectroscopy analysis was measured by Nexus 870 FT-IR. X-ray photoelectron spectroscopy (XPS) was performed with Escalab 250xi.

\subsection{Quality assurance/quality control (QA/QC)}

The developed method, analysis trace COPs in water using NPCs-coated fibers, was evaluated with linearity, sensitivity, recovery, precision and reproducibility according to QA/QC protocols. $^{33,34}$ Linearity was performed using seven different initial concentrations. Sensitivity was presented by limits of detection (LODs) and limits of quantification (LOQs). After spiked analyte into ultra-pure water with minimum detectable concentration $(n=6)$, LODs and LOQs were calculated on the basis of signal-to-noise ratio of $3(\mathrm{~S} / \mathrm{N}=3)$ and $10(\mathrm{~S} / \mathrm{N}=10)$, respectively. Recoveries of the proposed method were calculated using the ratio of the peak areas of the spiked samples of known concentration of COPs to those of spiked aqueous solutions. Precision was estimated in terms of relative standard deviations (RSDs) of intra-day and inter-day using three replicates of samples. The fiber-to-fiber reproducibility was evaluated using five fibers. Analytical procedure was composed of sampling, extracting and quantifying. Concentrations of standards, calibration step, repeatability of results and recovery determination (trueness) were considered as main contributions for expanded uncertainty. ${ }^{35}$ The detailed calculation for measurement uncertainties by the bottom-up method was summarized in Table S1. $\dagger$

\section{Results and discussion}

\subsection{Characterization of the nanoporous carbons}

The porosity structure of NPCs was analyzed by $\mathrm{N}_{2}$ adsorption/ desorption isothermal curves. In Fig. 1a, an I/IV-type adsorption isothermal curve was shown, indicated the NPCs were composed by both micropore and mesopore. It was further confirmed by the pore size distributions (PSDs), in Fig. 1b. All of NPCs exhibited a large scale of pore size with multimodal PSD. Micropore has mainly centered at $0.80 \mathrm{~nm}$ and $0.19 \mathrm{~nm}$. Mesopore has mainly centered at 5-10 nm. From about $0.80 \mathrm{~nm}$, the amounts of pores of NPC-8 were all higher than others, and NPC-4 > NPC-2 > NPC-0. Compared with previous reported activated NPCs, which typically possesses pores of $2 \mathrm{~nm}$ or smaller, the average pore sizes in NPCs were from 2 to $4.6 \mathrm{~nm}$, which can improve mass transfer for organic pollutants. The textural properties of NPCs from OSRS were summarized in Table 1.

The specific surface areas were ranged from 239 to $1253 \mathrm{~m}^{2}$ $\mathrm{g}^{-1}$, which were high that could result in strong surface adsorption energy and provide a tremendous adsorption interface for organic pollutants accumulation. The total pore volumes of NPCs were found to be ranged from 0.14 to $0.71 \mathrm{~cm}^{3}$ $\mathrm{g}^{-1}$. It can be seen that both specific surface area and total pore volume have been extended with increasing the addition amounts of the $\mathrm{KHCO}_{3}$, and the yields of NPCs were continuing slump. The micropore volumes were ranged from 0.1 to 0.54 $\mathrm{cm}^{3} \mathrm{~g}^{-1}$ and the mesopore volumes were ranged from 0.04 to $0.17 \mathrm{~cm}^{3} \mathrm{~g}^{-1}$. The mesopore/total pore volume ratios were found to be from $19 \%$ to $29 \%$. High volumes of mesopore were more favorable for rapid mass transfer of organic pollutants during extraction and desorption. In addition, the appropriate proportion of micropore guaranteed their high specific surface area for provide absorb sites and energy. Such NPCs with high specific surface area and wide pore size distribution were not only extremely beneficial for analysis chemistry of extraction on both high-rate and high-accumulation performance, but also extendable to the field in energy and gas storage.

NPC-8 was chosen to explore the architecture of NPCs by SEM. As shown in Fig. S2a and S2b, $\uparrow$ before grind, there were lots of inherent ordered lattice-like structure internally, which is good for mass transfer and could further generate higher specific surface area for more storage sites and stronger adsorption energy. A lot of disorder distributed micro/meso/ macropores were shown in Fig. S2c and S2d. $\dagger$ Moreover, Fig. S2d $\uparrow$ showed some graphene fringe clearly, which could induce the $\pi-\pi$ stack interaction between NPCs and organic pollutions. In general, thus porosity structure is desirable for applied in SPME technology.

TG and DSC analysis were used to study the reactions during activation and carbonization process and evaluate thermal stability of the NPCs (Fig. S3†). Heating the mixture of the hydrochar and $\mathrm{KHCO}_{3}$ from 30 to $1000{ }^{\circ} \mathrm{C}$ under $\mathrm{N}_{2}$ protection. At about $200{ }^{\circ} \mathrm{C}$, a sheer weight loss and a well-marked peak in DSC curve were appeared, which corresponds to the decomposition of $\mathrm{KHCO}_{3}$ to potassium carbonate $\left(\mathrm{K}_{2} \mathrm{CO}_{3}\right)$ alone with endothermic process $\left(2 \mathrm{KHCO}_{3} \rightarrow \mathrm{K}_{2} \mathrm{CO}_{3}+\mathrm{CO}_{2}+\mathrm{H}_{2} \mathrm{O}, \Delta H<\right.$ $0) .{ }^{24}$ From 200 to $700{ }^{\circ} \mathrm{C}$, approximately, weight loss rate was gentle, which is attributed to slow decomposition of the hydrochar. Importantly, with the increased temperature $(>700$ ${ }^{\circ} \mathrm{C}$ ), another sheer weight loss and observably DSC peak were present, which may be result from the decomposition of $\mathrm{K}_{2} \mathrm{CO}_{3}$ and the redox reactions between $\mathrm{K}_{2} \mathrm{CO}_{3}$ and the hydrochar or potassium oxide and the hydrochar $\left(\mathrm{K}_{2} \mathrm{CO}_{3} \rightarrow \mathrm{K}_{2} \mathrm{O}+\mathrm{CO}_{2}, \Delta H<\right.$ $0 ; \mathrm{K}_{2} \mathrm{CO}_{3}+2 \mathrm{C} \rightarrow 2 \mathrm{~K}+3 \mathrm{CO}, \Delta H<0 ; \mathrm{K}_{2} \mathrm{O}+\mathrm{C} \rightarrow 2 \mathrm{~K}+\mathrm{CO}, \Delta H<$ $0) .{ }^{24}$ During these reactions, carbon monoxide was produced due to the oxidization by $\mathrm{K}_{2} \mathrm{CO}_{3}$ and formed a lots of nanopores by blowing. Potassium oxide and metallic potassium were embedding in carbon material, which generates nanopore after acid wash. In contrast, weight loss of acid washed NPCs were gentle from 100 to $1000{ }^{\circ} \mathrm{C}$. At around $100{ }^{\circ} \mathrm{C}$, there was a marked weight loss and DSC endothermic peak due to the loss of free water. And, up to $400{ }^{\circ} \mathrm{C}$, its weight loss was inconspicuous, which implied great thermal stability for SPME fiber.

These partly graphitic amorphous-like structure of the NPCs were also evidenced by XRD and Raman spectroscopy. XRD patterns of the NPCs derived from different mixed mass ratio (Fig. S4†) shown a tiny characteristic peak at $2 \theta=26^{\circ}$ 

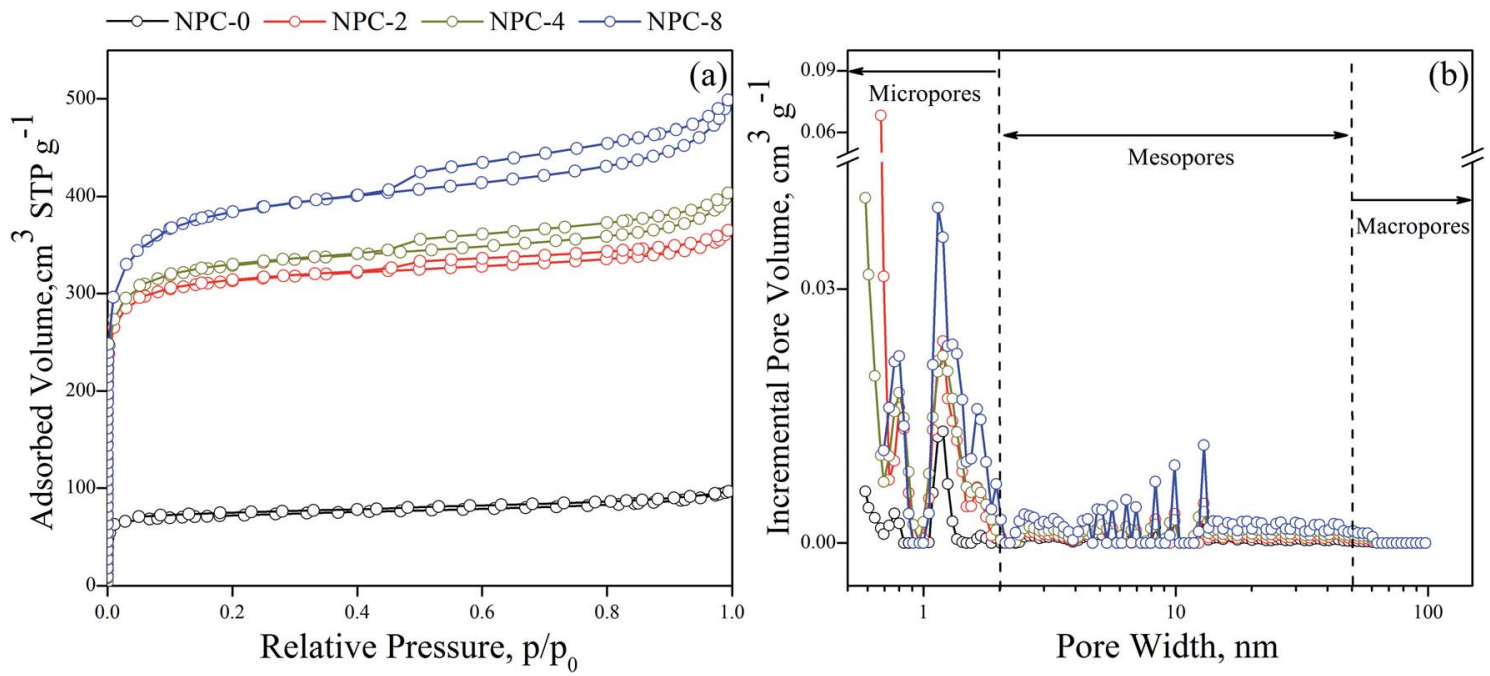

Fig. 1 Nitrogen adsorption-desorption isotherm (a) and pore size distribution (b) of the NPCs.

Table 1 Textural properties of the NPCs generated from OSRS via green $\mathrm{KHCO}_{3}$ chemical activation

\begin{tabular}{llllll}
\hline Material & Yield (\%) & $S_{\text {BET }}\left(\mathrm{m}^{2} \mathrm{~g}^{-1}\right)$ & $V_{\mathrm{p}}^{a}\left(\mathrm{~cm}^{3} \mathrm{~g}^{-1}\right)$ & $V_{\text {micro }}^{b}\left(\mathrm{~cm}^{3} \mathrm{~g}^{-1}\right)$ & 0.1 \\
NPC-0 & 48 & 239 & 0.14 & 0.43 & $0.04(29)$ \\
NPC-2 & 47 & 1009 & 0.54 & 0.47 & $0.11(20)$ \\
NPC-4 & 40 & 1063 & 0.58 & 0.54 & $0.11(19)$ \\
NPC-8 & 35 & 1253 & 0.71 & $\left.\mathrm{~cm}^{3} \mathrm{~g}^{-1}\right)$ \\
\hline
\end{tabular}

${ }^{a}$ Pore volume at $P / P_{0}=0.95 .{ }^{b}$ Micropore volume was determined by applying the non-local density functional theory (NLDFT). ${ }^{c}$ Mesopore volume was obtained by the difference between pore volume and micropore volume; the percentage of pore volume that corresponds to the mesopores was given in parentheses.

corresponding to the (002) plane and a remarkable characteristic peak at $2 \theta=44^{\circ}$ to the (101) plane which belong to the hexagonal graphite (JCPDS card no. 41-1487). ${ }^{36}$

Meanwhile, the Raman spectrums of the NPCs derived from different mixed mass ratios (Fig. S5 $\dagger$ ) displayed a G-band at about $1571 \mathrm{~cm}^{-1}$ corresponding to $\mathrm{sp}^{2} \mathrm{C}$ from graphitic layers and a D-band at about $1345 \mathrm{~cm}^{-1}$ corresponding to defective graphitic structure. Further, $I_{\mathrm{G}} / I_{\mathrm{D}}\left(I_{\mathrm{D}}\right.$ represents the intensity of the $\mathrm{D}$ band, and $I_{\mathrm{G}}$ stands for the intensity of the $\mathrm{G}$ band) ranged from 1.07 to 1.16 indicated partly graphitization of NPCs and changed with different addition ratio of the $\mathrm{KHCO}_{3}$. Among them, NPC-8 was lowest that suggested a lower graphitic with more defective structure. It seems that the moderate amount of activator would leading to form a more graphitic or defective structure. Graphitization structure would induce $\pi-\pi$ stack interaction with organic pollutants. And, defective structure would avoid the lamellar gapless stacking and form more nanopores. These NPCs with part graphitization and defect are not only expected electrode materials ${ }^{37}$ because of their high conductivities, but also extremely desirable for the application as SPME fibers due to their great extraction capacity.

FTIR spectroscopic analysis was used to evaluate the surface nature of the NPCs (Fig. S6†). There was no any remarkable peak in all of them, indicated the process of activating and carbonizing at high temperature could remove lots of various surface functional groups, which is consisted with previous reports. ${ }^{30}$ However, thus carbon materials are more suitable for SPME technology because of increased physio-chemical stability. Taken NPC-4 for example to probe the elemental composition of surface and chemical states of carbon and nitrogen species by XPS analysis. As shown in XPS spectrum (Fig. S7a†), the characteristic peaks of carbon, oxygen, nitrogen and silicon were appeared, and the contents ratio are $88.61 \%$, $9.56 \%, 1.16 \%$, and $0.67 \%$, respectively, which exhibited that inherent impurities and potassium salt produced during activating and carbonizing had been removed through the acid etching process. The high-resolution C 1s spectra (Fig. S7b †) revealed that the carbon peak can be resolved into five main peaks: $\mathrm{C}=\mathrm{C}\left(\mathrm{sp}^{2} \mathrm{C}, 284.5 \mathrm{eV}, 29.3 \%\right), \mathrm{C}-\mathrm{C}\left(\mathrm{sp}^{3} \mathrm{C}, 284.9 \mathrm{eV}\right.$, $30.8 \%$ ), C-O (epoxy/esters, $285.7 \mathrm{eV}, 15.0 \%$ ), $\mathrm{C}=\mathrm{O}$ (ketonic, $286.8 \mathrm{eV}, 8.1 \%$ ) and $\mathrm{O}=\mathrm{C}-\mathrm{O}$ (carboxylate, $289.2 \mathrm{eV}, 16.9 \%)^{38}$ Abundant $\mathrm{sp}^{2} \mathrm{C}$, resulted from partly graphene, could increase extraction capacity of the NPCs to aromatic compounds because of $\pi-\pi$ stacking interaction. ${ }^{11}$

As shown in Fig. S7c, $\dagger$ the high-resolution $\mathrm{N}$ 1s spectra was fitted by four peaks which located at $398.0 \mathrm{eV}$ attributed to pyridinic-N (N-6), $399.7 \mathrm{eV}$ due to pyrrolic-N (N-5), $400.8 \mathrm{eV}$ from quaternary-N (N-Q) and 402.5 because of oxidized-N (N-X).$^{37}$ And 
their represent ratio were $11.1 \%, 41.4 \%, 32.6 \%$ and $14.9 \%$, respectively. It exposed that nitrogen-containing compounds of OSRS, such as amino group, were mainly conversed into N-5 and N-Q during the activation and carbonization process. ${ }^{38}$ Some defected space of the inter wall which formed from part $\mathrm{N}$ 5 and N-6 could enhance mass transfer and provide additional storage sites for organic pollutants. ${ }^{39,40}$ Thus nitrogen doped carbon material also could be further considered as ultra-high capacity battery anodes and supercapacitors in the future. ${ }^{37}$

\subsection{Evaluations of the nanoporous carbon-coated fibers}

3.2.1. Surface morphology. As seen in Fig. 2a, taken NPC-8coated fiber as example, NPC-8 was homogeneous distributed on the surface of stainless steel wire. And, the thickness of NPC8 coating was about $16.5 \mu \mathrm{m}$, because the diameter of the NPC8-coated fiber was $160 \mu \mathrm{m}$ and the stainless steel wire used as the supporting substrate was $127 \mu \mathrm{m}$. From Fig. 2b, the synthesized NPC-8 was disordered stacked and formed many crevices, further increased the amounts of macropore and enhanced mass transfer rate. Thus, pile up also increased specific surface area and total pore volume.

3.2.2. Extraction efficiency of the nanoporous carboncoated fibers. The extraction capacities of NPC-coated fibers for CBs and PCBs were compared with the most frequently used commercial PDMS fiber under the same conditions. As shown in Fig. 3a, the extraction efficiencies of all of NPC-coated fibers were higher than those of the commercial PDMS fiber (2-38 times), especially for lower chlorinated benzene. These NPCcoated fibers also showed excellent extraction performance for PCBs, as shown in Fig. 3b, which were about 1-3 times higher than PDMS fiber. Same as CBs, the gaps of extraction efficiencies between NPC-coated fibers and PDMS fiber were gradually decreased with higher chlorinated biphenyls, which indicated NPCs are more suitable for extraction low chlorinated volatile organic compounds. It is may be result from the porosity structure manly composed of micropore that has no capacity to fasten relative large size organic pollutants and the increased extraction capacity of PDMS because of more similar polarity with higher chlorinated.

Among these NPC-coated fibers, NPC-8 fiber was the most efficient for the extraction of selected CBs and PCBs. NPC-8 >
NPC-2 > NPC-4 > NPC-0 and NPC-8 > NPC-2 > NPC-0 $\approx$ NPC-4 were found at lower and higher COPs, respectively. These results can be explained with pore size distribution, partly graphene, partly defect and specific surface area.

As we known, higher specific surface area is corresponding to higher extraction capacity because of higher surface interaction sites and stronger surface absorb energy. Meanwhile, high ratio of mesopore to total pore was crucial for providing available inner surface area and micropore because mesopore has controlled the mass transfer of COPs. ${ }^{20}$ Both partly graphitization and defect has contributed to enhance extraction capacity. Therefore, in general, activation with appropriate amounts of $\mathrm{KHCO}_{3}$ was necessary. What is more, it can be found that same molecular formula with different structure also do some influences on extraction efficiency.

\subsection{Environmental applications}

3.3.1. Optimizations. Before developing a new method to analysis trace COPs from water, NPC-8-coated fiber was chosen, and desorption temperature, extraction temperature, and extraction time were optimized (Fig. $\mathrm{S} 8 \dagger$ ). As shown in Fig. S8-1a and $b, \uparrow$ the extracted COPs using NPC-8-coated fiber were released totally under $290{ }^{\circ} \mathrm{C}$ for $5 \mathrm{~min}$. Remarkably, the extraction efficiencies of NPC-8-coated fiber have been significant increased under high extraction temperature (Fig. S8-2a and $\mathrm{b}_{\dagger} \dagger$ ), which was different with previous studies. ${ }^{10,32}$ It is may because that the strong adsorption force for COPs onto NPC-8-coated fiber could avoid the desorption process with increased diffusion constant from NPC-8-coated fiber under high temperature. Hence, it was a dazzling highlight for NPC-8coated fiber in SPME, more suitable to apply in situ. Moreover, it can be seen that, for HCB, the equilibrium time was about $60 \mathrm{~min}$ (Fig. S8-3a and $\mathrm{b}_{\dagger} \dagger$ ). For others, the equilibrium time was about $25 \mathrm{~min}$. Considering the analysis time of GC-ECD (24.5 min), 25 min was chosen as extraction time for further study.

3.3.2. Evaluation of analytical performance. The evaluation of proposed method for the analysis of COPs in water using NPC-8-coated fiber included linear range, coefficient of determination $\left(\mathrm{R}^{2}\right)$, LODs, LOQs and repeatability was presented in Table 2. All of selected CBs and PCBs showed great linearity $\left(\mathrm{R}^{2}\right.$ from 0.9991 to 0.9998 ) at concentrations in the range of 5-

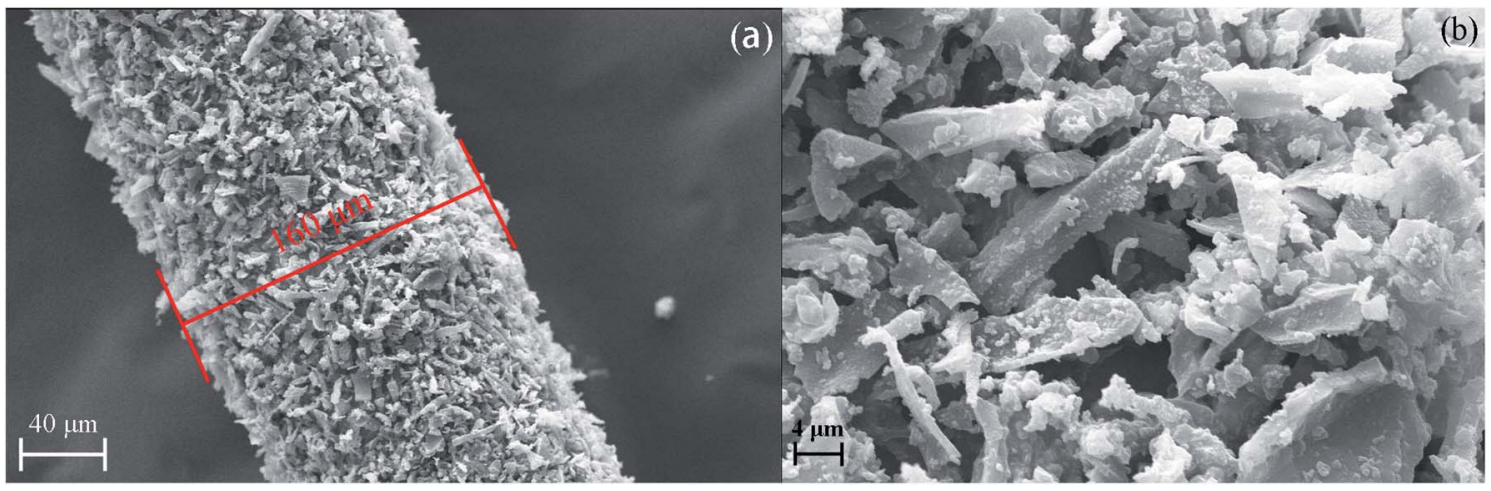

Fig. 2 Typical SEM images from NPC-8-coated fiber (a) 800x, (b) 5000×. 


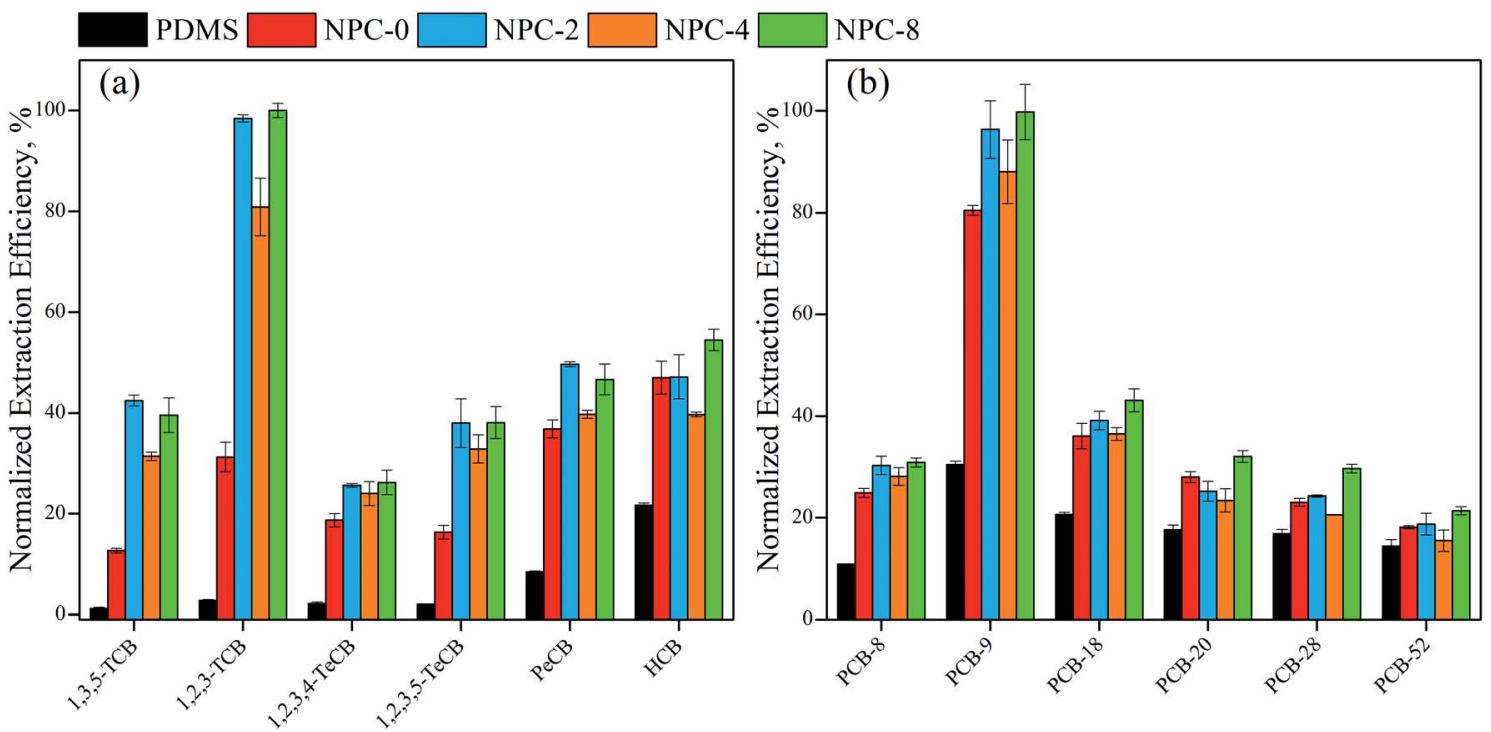

Fig. 3 Comparison of the extraction capacity of the NPCs-coated fibers and commercial PDMS fiber for the CBs (a) and PCBs (b). Concentration of target analytes (100 ng L ${ }^{-1}$ for 1,2,3,4-TeCB, PCB-20, PCB-28, PCB-52; 250 ng L $^{-1}$ for PCB-8, PCB-18; 500 ng L ${ }^{-1}$ for 1,3,5-

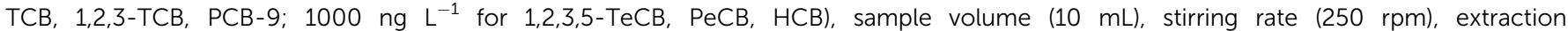
temperature $\left(60{ }^{\circ} \mathrm{C}\right)$, extraction time $(25 \mathrm{~min})$, desorption temperature $\left(290{ }^{\circ} \mathrm{C}\right)$ and desorption time $(5$ min) Normalized extraction efficiencies $(\%)=\frac{\text { Peak area of each analyte }}{\text { maximum peak area }} \times 100$.

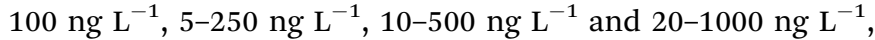
respectively. The LODs were from 0.08 to $0.64 \mathrm{ng} \mathrm{L}^{-1}$. The LOQs were from 0.27 to $2.13 \mathrm{ng} \mathrm{L}^{-1}$. RSDs of intra-day and inter-day with a single fiber were in the range of $2.63-6.73 \%$ and $2.22-$ $7.12 \%$, respectively, which display a good precision. The RSDs of fiber-to-fiber were below $6.41 \%$. In Table $\mathrm{S} 2, \dagger$ compared with other reported methods, LODs of this method by NPC-8-coated fiber were low, which exhibited excellent sensitivity. Besides, after underwent 200 extraction/desorption cycles with a single fiber, the extraction capacity was unchanged, indicated the excellent thermal stability and mechanical strength of prepared NPCs coated-fibers. On the whole, this method was exceedingly suitable to determine trace level of COPs in water.
3.3.3. Real sample analysis. In order to further evaluate the recoveries of the method in real environment sample, the surface water of Taihu lake (Jiangsu, China) was collected and filtered using $0.45 \mu \mathrm{m}$ membrane. As shown in Table 3, 1,2,3,4TeCB (0.45 ng L $\left.{ }^{-1}\right)$, PeCB (8.32 ng L ${ }^{-1}$ ), HCB (5.88 ng L $\left.{ }^{-1}\right)$, PCB-

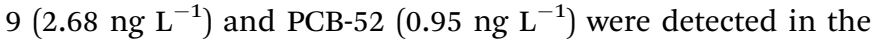
surface water of Taihu lake. Good recoveries were obtained from $86.66 \%$ to $103.27 \%$ by spiking twelve selected CBs and PCBs

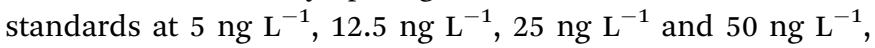
respectively. Moreover, partly detected COPs in this study were compared with other water samples in the literatures (Table S3†), showed that Taihu lake was slightly polluted. Besides, the advantages and drawbacks of the diverse analytical

Table 2 Analytical performance of the NPC-8-coated fiber

\begin{tabular}{|c|c|c|c|c|c|c|c|c|c|}
\hline \multirow[b]{2}{*}{ Analytes } & \multicolumn{4}{|c|}{ Regression line } & \multirow{2}{*}{$\begin{array}{l}\text { LODs } \\
\left(\operatorname{ng~L~}^{-1}\right)\end{array}$} & \multirow{2}{*}{$\begin{array}{l}\text { LOQs } \\
\left(\mathrm{ng} \mathrm{L}^{-1}\right)\end{array}$} & \multicolumn{3}{|l|}{$\mathrm{RSD}(\%)$} \\
\hline & Slope & Intercept & $R^{2}$ & $\begin{array}{l}\text { Range } \\
\left(\mathrm{ng} \mathrm{L} \mathrm{L}^{-1}\right)\end{array}$ & & & $\begin{array}{l}\text { Intra-day } \\
(n=3)\end{array}$ & $\begin{array}{l}\text { Inter-day } \\
(n=3)\end{array}$ & $\begin{array}{l}\text { Fiber-to-fiber } \\
(n=5)\end{array}$ \\
\hline 1,3,5-ТСВ & $8.50 \times 10^{7}$ & $2.25 \times 10^{7}$ & 0.9994 & $10-500$ & 0.34 & 1.15 & 2.63 & 3.65 & 5.04 \\
\hline $1,2,3-\mathrm{TCB}$ & $1.50 \times 10^{8}$ & $2.00 \times 10^{8}$ & 0.9993 & $10-500$ & 0.19 & 0.64 & 4.36 & 2.22 & 4.28 \\
\hline $1,2,3,4$-ТеСВ & $4.00 \times 10^{8}$ & $1.20 \times 10^{8}$ & 0.9996 & 5-100 & 0.09 & 0.30 & 3.80 & 3.20 & 2.17 \\
\hline 1,2,3,5-ТеСВ & $3.40 \times 10^{7}$ & $9.00 \times 10^{9}$ & 0.9994 & 20-1000 & 0.28 & 0.92 & 3.86 & 4.74 & 2.31 \\
\hline PeCB & $6.00 \times 10^{7}$ & $1.50 \times 10^{8}$ & 0.9997 & 20-1000 & 0.64 & 2.13 & 6.73 & 4.25 & 5.89 \\
\hline HCB & $8.00 \times 10^{7}$ & $9.00 \times 10^{7}$ & 0.9993 & 20-1000 & 0.30 & 1.00 & 4.96 & 7.12 & 6.41 \\
\hline PCB-8 & $8.00 \times 10^{7}$ & $4.00 \times 10^{7}$ & 0.9996 & $5-250$ & 0.29 & 0.95 & 4.35 & 5.07 & 5.32 \\
\hline PCB-9 & $1.30 \times 10^{8}$ & $1.00 \times 10^{8}$ & 0.9995 & $10-500$ & 0.22 & 0.75 & 4.23 & 2.31 & 4.47 \\
\hline PCB-18 & $1.00 \times 10^{8}$ & $9.00 \times 10^{7}$ & 0.9997 & $5-250$ & 0.21 & 0.70 & 4.05 & 4.52 & 2.96 \\
\hline PCB-20 & $2.00 \times 10^{8}$ & $6.00 \times 10^{7}$ & 0.9998 & $5-100$ & 0.08 & 0.27 & 4.57 & 5.26 & 2.33 \\
\hline PCB-28 & $2.00 \times 10^{8}$ & $1.00 \times 10^{8}$ & 0.9991 & $5-100$ & 0.13 & 0.45 & 5.23 & 4.71 & 3.96 \\
\hline PCB-52 & $1.20 \times 10^{8}$ & $4.00 \times 10^{8}$ & 0.9997 & 5-100 & 0.12 & 0.41 & 2.94 & 3.64 & 4.88 \\
\hline
\end{tabular}


Table 3 Results for the determination of the CBs and PCBs in Taihu lake using developed method

\begin{tabular}{|c|c|c|c|c|}
\hline Analytes & Concentration $\left(\mathrm{ng} \mathrm{L}^{-1}\right)$ & Spiked $\left(\mathrm{ng} \mathrm{L}^{-1}\right)$ & Detected $\left(\mathrm{ng} \mathrm{L^{-1 }}\right)$ & Recovery $(\%, n=3)$ \\
\hline $1,3,5-\mathrm{TCB}$ & $\mathrm{nd}^{a}$ & 25.00 & $24.41 \pm 1.95$ & $97.64 \pm 0.02$ \\
\hline $1,2,3$-TCB & nd & 25.00 & $24.11 \pm 2.65$ & $96.44 \pm 0.03$ \\
\hline $1,2,3,5-\mathrm{TeCB}$ & nd & 50.00 & $47.56 \pm 4.71$ & $95.12 \pm 0.05$ \\
\hline PeCB & $8.32 \pm 0.67$ & 50.00 & $58.33 \pm 3.50$ & $100.02 \pm 0.04$ \\
\hline HCB & $5.88 \pm 0.41$ & 50.00 & $57.52 \pm 6.33$ & $103.27 \pm 0.06$ \\
\hline PCB-18 & nd & 12.50 & $12.42 \pm 1.04$ & $99.37 \pm 0.01$ \\
\hline PCB-20 & nd & 5.00 & $4.33 \pm 0.41$ & $86.66 \pm 0.00$ \\
\hline PCB-28 & nd & 5.00 & $4.80 \pm 0.60$ & $96.07 \pm 0.01$ \\
\hline PCB-52 & $0.95 \pm 0.10$ & 5.00 & $6.06 \pm 0.73$ & $102.23 \pm 0.01$ \\
\hline
\end{tabular}

methodology used to determine COPs in water were summarized in Table $\mathrm{S} 4, \uparrow$ indicated proposed method was advanced and could play an important role in future.

\section{Conclusion}

In conclusion, our study indicated that crop waste could be sustainable transformed into a series of partly graphitic, amorphous-like NPCs with huge specific surface area and large pore volume by the green process of hydrothermal reaction and $\mathrm{KHCO}_{3}$ activation. And, self-made SPME fibers could be fabricated using NPCs by means of sol-gel method. Owing to the strong solid surface adsorption energy, abundantly available storage sites, hydrophobic and $\pi-\pi$ interaction, NPCs-coated fibers exhibited higher extraction capacities than PDMS fiber for the COPs, which indicated NPCs-coated fibers could be extended to microextraction other organic pollutants with high performance in future. In this study, coupled with GC-ECD, an excellent analytical method with great linearity, sensitivity, and repeatability for trace COPs in water was developed. And, it had advantages of easy preparation, low cost, and long life-span (more than 200 extraction/desorption cycles). The findings provide new opportunities for the sustainable conversion of crop-waste into NPCs for SPME.

\section{Conflicts of interest}

There are no conflicts to declare.

\section{Acknowledgements}

This study was financially supported by the National Key Basic Research Program of China [2014CB441105], National Science and Technology Major Project of China [2017YFD0800704], the Outstanding Youth Fund of the Natural Science Foundation of Jiangsu, China [BK20150050], the National Natural Science Foundation of China [41671236], and Key Program of Frontier Sciences, Chinese Academy of Sciences [QYZDJ-SSW-DQC035].

\section{References}

1 C. L. Arthur and J. Pawliszyn, Anal. Chem., 1990, 62, 21452148.

2 F. X. Wang, J. Zheng, J. L. Qiu, S. Q. Liu, G. S. Chen, Y. X. Tong, F. Zhu and G. F. Ouyang, ACS Appl. Mater. Interfaces, 2017, 9, 1840-1846.

3 C. H. Xu, G. S. Chen, Z. H. Xiong, Y. X. Fan, X. C. Wang and Y. Liu, TrAC, Trends Anal. Chem., 2016, 80, 12-29.

4 J. Q. Xu, G. S. Chen, S. Y. Huang, J. L. Qiu, R. F. Jiang, F. Zhu and G. F. Ouyang, TrAC, Trends Anal. Chem., 2016, 85, 26-35.

5 É. A. Souza-Silva, N. Reyes-Garcés, G. A. Gómez-Ríos, E. Boyacı, B. Bojko and J. Pawliszyn, TrAC, Trends Anal. Chem., 2015, 71, 249-264.

6 N. Reyes-Garces, E. Gionfriddo, G. A. Gomez-Rios, M. N. Alam, E. Boyaci, B. Bojko, V. Singh, J. Grandy and J. Pawliszyn, Anal. Chem., 2018, 90, 302-360.

7 H. Piri-Moghadam, M. N. Alam and J. Pawliszyn, Anal. Chim. Acta, 2017, 984, 42-65.

8 M. J. Trujillo-Rodriguez, H. Nan and J. L. Anderson, J. Chromatogr. A, 2018, 1540, 11-20.

9 X. F. Guo, A. Mulati, M. Wu, J. Zhang, L. Yang, F. Q. Zhao and B. Z. Zeng, RSC Adv., 2017, 7, 22256-22262.

10 S. Q. Liu, Q. K. Hu, J. Zheng, L. J. Xie, S. B. Wei, R. F. Jiang, F. Zhu, Y. Liu and G. F. Ouyang, J. Chromatogr. A, 2016, 1450, 9-16.

11 S. Q. Liu, D. R. Chen, J. Zheng, L. W. Zeng, J. J. Jiang, R. F. Jiang, F. Zhu, Y. Shen, D. C. Wu and G. F. Ouyang, Nanoscale, 2015, 7, 16943-16951.

12 S. Ansari and M. Karimi, Talanta, 2017, 164, 612-625.

13 L. Chen and X. J. Huang, Analyst, 2017, 142, 4039-4047.

14 P. Rocio-Bautista, I. Pacheco-Fernandez, J. Pasan and V. Pino, Anal. Chim. Acta, 2016, 939, 26-41.

15 F. Y. Lv, N. Gan, J. L. Huang, F. T. Hu, Y. T. Cao, Y. Zhou, Y. R. Dong, L. Zhang and S. Jiang, Microchim. Acta, 2017, 184, 2561-2568.

16 X. Y. Song, J. Chen and Y. P. Shi, TrAC, Trends Anal. Chem., 2017, 86, 263-275. 
17 Y. Liu, Y. F. Huang, G. S. Chen, J. L. Huang, J. Zheng, J. Q. Xu, S. Q. Liu, J. L. Qiu, L. Yin, W. H. Ruan, F. Zhu and G. F. Ouyang, Anal. Chim. Acta, 2018, 1015, 20-26.

18 M. Ghaemmaghami, Y. Yamini, H. Amanzadeh and B. H. Monjezi, Chem. Commun., 2018, 54, 507-510.

19 J. Zheng, J. L. Huang, F. Xu, F. Zhu, D. C. Wu and G. F. Ouyang, Nanoscale, 2017, 9, 5545-5550.

20 J. Zheng, K. Wang, Y. R. Liang, F. Zhu, D. C. Wu and G. F. Ouyang, Chem. Commun., 2016, 52, 6829-6832.

21 H. Nishihara and T. Kyotani, Adv. Mater., 2012, 24, 44734498.

22 J. H. Hou, C. B. Cao, F. Idrees and X. L. Ma, ACS Nano, 2015, 9, 2556-2564.

23 J. C. Wang and S. Kaskel, J. Mater. Chem., 2012, 22, 2371023725.

24 M. Sevilla and A. B. Fuertes, ChemSusChem, 2016, 9, 18801888.

25 C. Wang, M. J. O'Connell and C. K. Chan, ACS Appl. Mater. Interfaces, 2015, 7, 8952-8960.

26 L. Q. Ji, Renewable Sustainable Energy Rev., 2015, 44, 561-575. 27 Q. L. Ma, L. J. Han and G. Q. Huang, Bioresour. Technol., 2017, 234, 53-60.

28 D. B. Wei, T. Kameya and K. Urano, Environ. Int., 2007, 33, 894-902.

29 M. Y. Yao, T. T. Hu, Y. F. Wang, Y. J. Du, C. C. Hu and R. J. Wu, Environ. Pollut., 2017, 229, 837-845.
30 M. Sevilla, G. A. Ferrero and A. B. Fuertes, Carbon, 2017, 114, 50-58.

31 H. Cheng, Y. Song, Y. R. Bian, F. Wang, R. T. Ji, W. X. He, C. G. Gu, G. F. Ouyang and X. Jiang, Microchim. Acta, 2018, 185, 56.

32 S. Y. Li, F. Zhu, R. F. Jiang and G. F. Ouyang, J. Chromatogr. A, 2016, 1429, 1-7.

33 P. Konieczka and J. Namiesnik, J. Chromatogr. A, 2010, 1217, 882-891.

34 M. Papageorgiou, D. Lambropoulou, C. Morrison, J. Namiesnik and J. Plotka-Wasylka, Talanta, 2018, 183, 276-282.

35 N. Ratola, L. Santos, P. Herbert and A. Alves, Anal. Chim. Acta, 2006, 573-574, 202-208.

36 S. Hwang, S. Lee and J. S. Yu, Appl. Surf. Sci., 2007, 253, 56565659.

37 Z. Li, Z. Xu, X. Tan, H. Wang, C. M. B. Holt, T. Stephenson, B. C. Olsen and D. Mitlin, Energy Environ. Sci., 2013, 6, 871878.

38 B. H. Suryanto, S. Chen, J. Duan and C. Zhao, ACS Appl. Mater. Interfaces, 2016, 8, 35513-35522.

39 W. H. Shin, H. M. Jeong, B. G. Kim, J. K. Kang and J. W. Choi, Nano Lett., 2012, 12, 2283-2288.

40 F. C. Zheng, Y. Yang and Q. W. Chen, Nat. Commun., 2014, 5, 5261. 